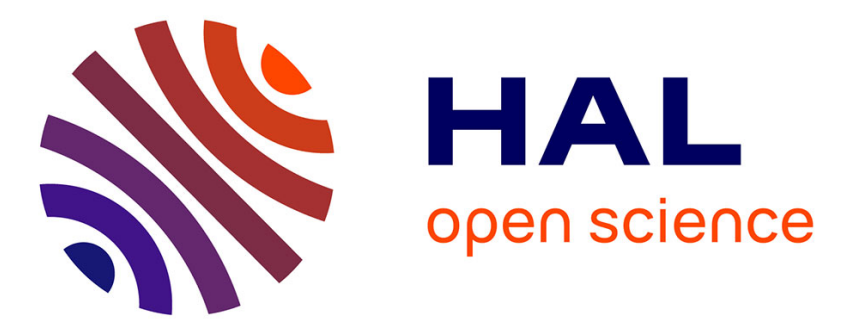

\title{
Topological properties of asymptotically stable sets
}

Emmanuel Moulay, Sanjay P. Bhat

\section{To cite this version:}

Emmanuel Moulay, Sanjay P. Bhat. Topological properties of asymptotically stable sets. Nonlinear Analysis: Theory, Methods and Applications, 2010, 73 (4), pp.1093-1097. 10.1016/j.na.2010.04.043 . hal-00551285

\section{HAL Id: hal-00551285 \\ https://hal.science/hal-00551285}

Submitted on 19 Jan 2021

HAL is a multi-disciplinary open access archive for the deposit and dissemination of scientific research documents, whether they are published or not. The documents may come from teaching and research institutions in France or abroad, or from public or private research centers.
L'archive ouverte pluridisciplinaire HAL, est destinée au dépôt et à la diffusion de documents scientifiques de niveau recherche, publiés ou non, émanant des établissements d'enseignement et de recherche français ou étrangers, des laboratoires publics ou privés. 


\title{
Topological properties of asymptotically stable sets
}

\author{
Emmanuel Moulay* \\ Xlim (UMR-CNRS 6172) - Département SIC \\ Université de Poitiers - Bât. SP2MI \\ Bvd Marie et Pierre Curie - BP 30179 \\ 86962 Futuroscope Chasseneuil Cedex - France \\ Sanjay P. Bhat** \\ TCS Innovation Labs Hyderabad \\ Tata Consultancy Services Limited \\ Deccan Park, Hi Tech City \\ Madhapur, Hyderabad 500081 - India
}

\begin{abstract}
Topological properties of the domain of attraction for dynamical systems are investigated. The main purpose of this paper is to prove that a compact, asymptotically stable attractor of a dynamical system defined on a locally compact metric space is a deformation retract of its domain of attraction, in a weak sense that is made precise. Under additional local assumptions, the attractor can be shown to be a retract, a deformation retract, or a strong deformation retract. The well known result that the domain of attraction of an asymptotically stable equilibrium is contractible follows as a corollary.
\end{abstract}

Keywords: Asymptotically stable sets, domain of attraction, retraction, Lyapunov functions.

\section{Introduction}

In [1, Thm. 21], Eduardo Sontag has shown that the domain of attraction of a dynamical system with an asymptotically stable equilibrium point is contractible. In [2], it is proved that global asymptotic stability on a vector bundle implies contractibility of the base manifold. In particular, a continuous dynamical system on a state space that has the structure of a vector bundle on a compact manifold possesses no globally asymptotically stable equilibrium.

\footnotetext{
*(33) 549496855

** 91-40-6667-3561

Email addresses: emmanuel.moulay@univ-poitiers.fr (Emmanuel Moulay), sanjay@atc.tcs.com (Sanjay P. Bhat)

$U R L$ : https://bvent.univ-poitiers.fr/access/content/user/emoulay (Emmanuel Moulay)
} 
The main goal of this paper is to generalize Sontag's result [1, Thm. 21] for equilibria to compact, asymptotically stable sets. Our main result shows that a compact, asymptotically stable attractor of a continuous dynamical system defined on a locally compact metric space is a weak deformation retract of its domain of attraction in a sense that we define. A weak version of our result involving retract only has been stated in [3, Lemma 3.2]. We also show that, under additional topological assumptions, a weak deformation retract is a retract, a deformation retract, or a strong deformation retract. The additional assumptions are trivially satisfied in the case of an equilibrium, and hence $[1$, Thm. 21] follows as a corollary of our results.

We introduce the necessary notation and topological preliminaries in Section 2. Section 3 contains the main results. Finally, the conclusion is presented in Section 4 .

\section{Preliminaries}

Throughout this paper, we consider a continuous, time-invariant semi-flow $\varphi: \mathbb{R}_{\geq 0} \times \mathcal{M} \rightarrow \mathcal{M}$ on a locally compact topological space $\mathcal{M}$. The semi-flow $\varphi$ satisfies $\varphi(0, x)=x$ and $\varphi(t, \varphi(s, x))=\varphi(t+s, x)$ for all $t, s \in \mathbb{R}_{\geq 0}$ and $x \in \mathcal{M}$. The topological space $\mathcal{M}$, called the state space, and the semi flow $\varphi$, also called the evolution function, together define a dynamical system, which we denote by $(\mathcal{M}, \varphi)$ (see for instance [4] and [3]).

For each $t \in \mathbb{R}_{\geq 0}$, we let $\varphi_{t}$ denote the map $x \mapsto \varphi(t, x)$. Next, we recall the definition of stability.

Definition 1. The set $\mathcal{K} \subset \mathcal{M}$ is asymptotically stable if the following two conditions hold.

1. $\mathcal{K}$ is Lyapunov stable, that is, for every open neighborhood $\mathcal{V} \subseteq \mathcal{M}$ of $\mathcal{K}$, there exists an open neighborhood $\mathcal{U} \subseteq \mathcal{M}$ of $\mathcal{K}$ such that $\varphi_{t}(\overline{\mathcal{U}}) \subseteq \mathcal{V}$ for every $t \geq 0$.

2. $\mathcal{K}$ is attractive, that is, there exists an open neighborhood $\mathcal{W} \subseteq \mathcal{M}$ of $\mathcal{K}$ such that, for every $x \in \mathcal{W}$ and every open neighborhood $\mathcal{U} \subseteq \mathcal{M}$ of $\mathcal{K}$, there exists $T \geq 0$ such that $\varphi(t, x) \in \mathcal{U}$ for all $t>T$.

The domain of attraction of an asymptotically stable set $\mathcal{K} \subseteq \mathcal{M}$ is the set $\mathcal{A}$ of points $x$ such that, for every open neighborhood $\mathcal{U}$ of $\mathcal{K}$, there exists $T>0$ such that $\varphi(t, x) \in \mathcal{V}$ for all $t \geq T$.

It is well known that the domain of attraction $\mathcal{A}$ of a compact asymptotically stable set is open and invariant, that is, $\varphi_{t}(\mathcal{A})=\mathcal{A}$ for all $t \geq 0$.

Next, we recall various different notions of retracts. A survey on the theory of retracts can be founded in [5].

Definition 2. Let $X \subseteq Y \subseteq \mathcal{M}$.

1. $X$ is a retract of $Y$ if there exists a continuous map $r: Y \rightarrow X$, called a retraction, such that $r(x)=x$ for all $x \in X$. 
2. $X$ is a deformation retract of $Y$ if there exists a continuous homotopy $h:[0,1] \times Y \rightarrow \mathcal{M}$ such that

$$
\begin{aligned}
& h(0, y)=y, \\
& h(1, y) \in X, \\
& h(1, x)=x,
\end{aligned}
$$

for all $y \in Y$ and $x \in X$.

3. $X$ is a strong deformation retract of $Y$ if there exists a continuous homotopy $h:[0,1] \times Y \rightarrow \mathcal{M}$ such that

$$
\begin{aligned}
& h(0, y)=y, \\
& h(1, y) \in X, \\
& h(t, x)=x,
\end{aligned}
$$

for all $y \in Y, x \in X$ and $t \in[0,1]$.

4. $X$ is a weak deformation retract of $Y$ if every open neighborhood $U \subseteq \mathcal{M}$ of $X$ contains a strong deformation retract $V$ of $Y$ such that $X \subseteq V$.

A set $Y \subseteq \mathcal{M}$ is contractible if there exists $x \in \mathcal{M}$ such that the singleton set $X=\{x\}$ is a strong deformation retract of $Y$.

We will also need the following definition.

Definition 3. $X \subseteq \mathcal{M}$ is a neighborhood retract (respectively, neighborhood deformation retract, strong neighborhood deformation retract) of $\mathcal{M}$ if there exists an open neighborhood $Y \subseteq \mathcal{M}$ of $X$ such that $X$ is a retract (respectively, deformation retract, strong deformation retract) of $Y$.

Our first result relates definitions 2 and 3 .

Theorem 4. Suppose $X \subseteq \mathcal{M}$ is a weak deformation retract of $Y \subseteq \mathcal{M}$. Then the following statements hold.

1. If $X$ is a neighborhood retract of $\mathcal{M}$, then $X$ is a retract of $Y$.

2. If $X$ is a neighborhood deformation retract of $\mathcal{M}$, then $X$ is a deformation retract of $Y$.

3. If $X$ is a strong neighborhood deformation retract of $\mathcal{M}$, then $X$ is a strong deformation retract of $Y$.

Proof. First, note that since $X$ is a weak deformation retract of $Y$, for every neighborhood $U \subseteq \mathcal{M}$, there exist a set $V \subseteq U$ containing $X$, and a continuous map $h:[0,1] \times Y \rightarrow V$ such that $h(0, y)=y, h(1, y) \in V$ and $h(t, v)=v$ for all $y \in Y, v \in V$ and $t \in[0,1]$.

To prove 1), suppose $X$ is a neighborhood retract of $\mathcal{M}$. Then there exists an open neighborhood $U \subseteq \mathcal{M}$ of $X$ and a continuous map $s: U \rightarrow X$ such that $s(x)=x$ for all $x \in X$. Upon choosing the set $V$ and the homotopy $h$ as 
above, the map $r: Y \rightarrow X$ given by $r(y)=s(h(1, y))$ can easily be verified to be a retraction. Hence 1) follows.

Next,to prove 2), suppose $X$ is a neighborhood deformation retract of $\mathcal{M}$. Then there exists an open neighborhood $U \subseteq \mathcal{M}$ of $X$ and a continuous deformation retraction $s:[0,1] \times U \rightarrow X$ such that $s(0, u)=u, s(1, u) \in X$, and $s(1, x)=x$ for all $u \in U$ and $x \in X$. On choosing the set $V$ and the homotopy $h$ as in the first paragraph, it follows that the map $r:[0,1] \times Y \rightarrow X$ given by

$$
\begin{array}{rlrl}
r(t, y) & =h(2 t, y), & & t \in\left[0, \frac{1}{2}\right], y \in Y, \\
& =s(2 t-1, h(1, y)), & t \in\left(\frac{1}{2}, 1\right], y \in Y,
\end{array}
$$

is continuous and satisfies $r(0, y)=y, r(1, y) \in X$ and $r(1, x)=x$ for all $y \in Y$ and $x \in X$. Hence $X$ is a deformation retract of $Y$.

The proof of 3) follows from the proof of 2) above by noting that, in the case where $X$ is a strong neighborhood deformation retract of $\mathcal{M}$, the homotopy $s$ may be chosen to additionally satisfy $s(t, x)=x$ for all $x \in X$. Consequently, the homotopy $r$ constructed above will also satisfy $r(t, x)=x$ for all $x \in X$. Hence $X$ is a strong deformation retract of $Y$.

\section{Main result}

In this section, $\mathcal{M}$ is a locally compact metric space.

Theorem 5. Suppose $\mathcal{K}$ is a compact asymptotically stable set for the dynamical system $(\mathcal{M}, \varphi)$ with domain of attraction $\mathcal{A}$. Then $\mathcal{K}$ is a weak deformation retract of $\mathcal{A}$.

Proof. Because $\mathcal{K}$ is asymptotically stable, it follows from [3, Theorem 2.10] that there exists a continuous Lyapunov function $V: \mathcal{A} \rightarrow \mathbb{R}_{>0}$ such that $V(x)>0$ for all $x \in \mathcal{A} \backslash \mathcal{K}$ and $V(x)=0$ for all $x \in \mathcal{K}, V$ is proper, that is, $V^{-1}(B)$ is compact for every compact subset $B$ of $\mathbb{R}_{\geq 0}$, and $V$ is strictly decreasing along orbits of $\varphi$, that is, $V(\varphi(t, x))<V(x)$ for all $t>0$ and $x \in \mathcal{A} \backslash \mathcal{K}$.

For each $\rho>0$, define $\mathcal{U}_{\rho} \subseteq \mathcal{A}$ by

$$
\mathcal{U}_{\rho}=\{x \in \mathcal{A}: V(x) \leq \rho\} .
$$

The properties of $V$ imply that, for each $\rho>0, \mathcal{U}_{\rho}$ is compact and positively invariant, that is, $\varphi_{t}\left(\mathcal{U}_{\rho}\right) \subseteq \mathcal{U}_{\rho}$. Moreover, $\cap_{\rho>0} \mathcal{U}_{\rho}=\mathcal{K}$, so that for every open neighborhood $\mathcal{V} \subseteq \mathcal{M}$ of $\mathcal{K}$, there exists $\rho>0$ such that $\mathcal{U}_{\rho} \subseteq \mathcal{V}$.

Asymptotic stability implies that, for each $x \in \mathcal{A}$, there exists $T>0$ such that $\varphi(t, x) \in \mathcal{U}_{\rho}$ for all $t>T$. Hence, for each $\rho>0$ and $x \in \mathcal{A}$

$$
T_{\rho}(x)=\inf \left\{t \geq 0: \varphi(t, x) \in \mathcal{U}_{\rho}\right\}
$$

is well defined. It is clear that $T_{\rho}(x)=0$ for all $x \in \mathcal{U}_{\rho}$. 
We claim that $T_{\rho}: \mathcal{A} \rightarrow \mathbb{R}_{\geq 0}$ is continuous for each $\rho>0$. To prove our claim, let $\rho>0, \epsilon>0$ and consider a sequence $\left\{x_{n}\right\}_{n \in \mathbb{N}}$ converging to $x \in \mathcal{M}$. By the continuity of $V$, we have

$$
\lim _{n \rightarrow+\infty} V\left(\varphi\left(T_{\rho}(x)+\epsilon, x_{n}\right)\right)=V\left(\varphi\left(T_{\rho}(x)+\epsilon, x\right)\right)<\rho .
$$

Hence, the definition of $T_{\rho}$ implies that, for $n$ sufficiently large, we have $T_{\rho}\left(x_{n}\right) \leq$ $T_{\rho}(x)+\epsilon$. Similarly,

$$
\lim _{n \rightarrow+\infty} V\left(\varphi\left(T_{\rho}(x)-\epsilon, x_{n}\right)\right)=V\left(\varphi\left(T_{\rho}(x)-\epsilon, x\right)\right)>\rho
$$

leads to $T_{\rho}\left(x_{n}\right) \geq T_{\rho}(x)-\epsilon$ for $n$ sufficiently large. We conclude that $\mid T_{\rho}\left(x_{n}\right)-$ $T_{\rho}(x) \mid<\epsilon$ for all $n$ sufficiently large. Since $\varepsilon$ was chosen to be arbitrary, it follows that $\lim _{n \rightarrow+\infty} T_{\rho}\left(x_{n}\right)=T_{\rho}(x)$, and hence $T_{\rho}$ is continuous.

To show that $\mathcal{K}$ is a weak deformation retract of $\mathcal{A}$, choose an open neighborhood $\mathcal{V}$ of $\mathcal{K}$. Choose $\rho>0$ such that $\mathcal{U}_{\rho} \subseteq \mathcal{V}$. It is easy to verify that the map $h:[0,1] \times \mathcal{A} \rightarrow \mathcal{A}$ defined by $h(t, x)=\varphi\left(t T_{\rho}(x), x\right)$ is a continuous function satisfying $h(0, y)=y, h(1, y) \in \mathcal{U}_{\rho}$, and $h(t, x)=x$ for all $y \in \mathcal{A}, x \in \mathcal{U}_{\rho}$ and $t \in[0,1]$. Thus $\mathcal{U}_{\rho}$ is a strong deformation retract of $\mathcal{A}$. We have thus shown that the arbitrarily chosen neighborhood $\mathcal{V}$ of $\mathcal{K}$ contains a strong deformation retract of $\mathcal{A}$ that contains $\mathcal{K}$. Hence it follows that $\mathcal{K}$ is a weak deformation retract of $\mathcal{A}$.

Remark 6. Theorem 5 remains valid when $\mathcal{M}$ is a locally compact Hausdorff space and $\mathcal{K}$ is an invariant asymptotically stable set (see [4, Theorem 10.6]).

The following corollary of Theorem 5 follows in a straightforward manner from Theorem 4.

Corollary 7. Suppose $\mathcal{K}$ is a compact asymptotically stable set for the dynamical system $(\mathcal{M}, \varphi)$ with domain of attraction $\mathcal{A}$. Then the following statements hold.

1. If $\mathcal{K}$ is a neighborhood retract of $\mathcal{M}$, then $\mathcal{K}$ is a retract of $\mathcal{A}$.

2. If $\mathcal{K}$ is a neighborhood deformation retract of $\mathcal{M}$, then $\mathcal{K}$ is a deformation retract of $\mathcal{A}$.

3. If $\mathcal{K}$ is a strong neighborhood deformation retract of $\mathcal{M}$, then $\mathcal{K}$ is a strong deformation retract of $\mathcal{A}$.

Remark 8. Sets which are neighborhood retracts are rather common. In particular for finite dimensional spaces, neighborhood retracts are also Euclidean neighborhood retracts, which are well known and studied, for instance, in the appendices of [6, 7]. Finite $C W$ complex are Euclidean neighborhood retracts. Corollary 7 yields a necessary condition for the continuous stabilizability of neighborhood retracts. 
A point is always a strong neighborhood deformation retract if $\mathcal{M}$ is locally contractible. So, we recognize as a special case of Theorem 5 , the following result [1, Theorem 21] dedicated to contractibility of systems with asymptotically stable equilibrium point.

Corollary 9. Suppose that $\mathcal{M}$ is locally contractible. Let us consider the $d y$ namical system $(\mathcal{M}, d, \varphi)$ with an equilibrium point $x_{e}$. Suppose that $x_{e}$ is asymptotically stable. The domain of attraction, which is given by

$$
\mathcal{A}=\left\{x \in \mathcal{M}: \lim _{t \rightarrow+\infty} \varphi(t, x)=x_{e}\right\}
$$

is a contractible set.

In fact, the result [1, Theorem 21] remains true for general topological spaces due to the fact that the flow provides a natural homotopy.

The following proposition describes a common situation in which the set $\mathcal{K}$ of interest is a strong neighborhood deformation retract of $\mathcal{M}$.

Proposition 10. Suppose $\mathcal{M}$ is a smooth finite-dimensional manifold, and $\mathcal{K}$ is a compact, embedded submanifold of $\mathcal{M}$. Then $\mathcal{K}$ is a strong neighborhood deformation retract of $\mathcal{M}$.

Proof. Under the hypotheses on $\mathcal{M}$, there exists a tubular neighborhood of $\mathcal{K}$ [8, Thm. IV.5.1]. More precisely, there exists a vector bundle $\pi: \mathcal{E} \rightarrow \mathcal{K}$ over $\mathcal{K}$ with zero section $z: \mathcal{K} \mapsto \mathcal{E}$, an open neighborhood $\mathcal{V} \subseteq \mathcal{E}$ of $z(\mathcal{K})$, an open neighborhood $\mathcal{U} \subseteq \mathcal{M}$ of $\mathcal{K}$ and a diffeomorphism $g: \mathcal{U} \mapsto \mathcal{V}$ such that $g^{-1}(z(x))=x$ for all $x \in \mathcal{K}$. Since $\mathcal{K}$ is compact, we may choose $\mathcal{V}$ to further satisfy $t v \in \mathcal{V}$ for all $t \in[0,1]$ and all $v \in \mathcal{V}$. It is now easy to verify that the map $h:[0,1] \times \mathcal{U} \rightarrow \mathcal{U}$ defined by $h(t, x)=g^{-1}[(1-t) g(x)]$ is continuous and satisfies $h(0, y)=y, h(1, y) \in \mathcal{K}$ and $h(t, x)=x$ for all $y \in \mathcal{U}, x \in \mathcal{K}$ and $t \in[0,1]$. Thus $\mathcal{K}$ is a strong deformation retract of the open neighborhood $\mathcal{U}$, and the result follows.

Proposition 10 implies that a large class of invariant sets studied in automatic control are strong neighborhood deformation retracts. In [9], the authors prove that if $\mathcal{M}$ is a locally equiconnected set, then the property of being a neighborhood retract, a neighborhood deformation retract, or a strong neighborhood deformation retract are all equivalent.

If $\mathcal{K}$ is not a neighborhood retract, as Cantor or fractal sets (see for instance [10, Remark 5]), there is no practical necessary condition. Nevertheless, if we have no assumption on $\mathcal{K}$ we still have a theoretical result if the semi flow and the functions $T_{\rho}$ are uniformly continuous.

Proposition 11. Let us consider the dynamical system $(\mathcal{M}, d, \varphi)$ with a compact invariant asymptotically stable set $\mathcal{K}$ with domain of attraction $\mathcal{A}$ such that the semi flow $\varphi(t, x)$ is continuous in $x$ uniformly in $t$. If the function $T_{\rho}(x)$ given in (1) is also continuous in $x$ uniformly in $\rho$, then $\mathcal{K}$ is a strong neighborhood deformation retract of $\mathcal{A}$. 
Proof. For each $\rho>0$, define $h_{\rho}:[0,1] \times \mathcal{A} \rightarrow \mathcal{A}$ by $h_{\rho}(t, x)=\varphi\left(t T_{\rho}(x), x\right)$. By the same reasoning as in the proof of Theorem 5 , it can be shown that, for each $\rho>0, h_{\rho}$ yields a strong deformation retract from $\mathcal{A}$ onto $\mathcal{U}_{\rho}$.

Consider the family

$$
\mathcal{F}=\left\{h_{\rho}: \rho \leq 1\right\} .
$$

For each $t \in[0,1]$ and $x \in \mathcal{A}$

$$
\mathcal{F}(t, x)=\left\{h_{\rho}(t, x): \rho \leq 1\right\}
$$

is contained in the compact set $\mathcal{U}_{1}$ and hence has compact closure. Let us prove that the family $\mathcal{F}$ is equicontinuous. Let $x \in \mathcal{A}$ and $\epsilon>0$. Due to the assumption on the semi flow, there exists $\delta_{1}>0$ such that for all $\left|t_{1}-t_{2}\right|<\delta_{1}$ and all $y \in \mathcal{A}$ such that $d(x, y)<\delta_{1}$ we have $d\left(\varphi\left(t_{1}, x\right), \varphi\left(t_{2}, y\right)\right)<\epsilon$. Due to the assumption on $T_{\rho}(x)$, there exists $\delta_{2}$ such that for all $\left|t_{1}-t_{2}\right|<\delta_{2}$ and all $y \in \mathcal{A}$ such that $d(x, y)<\delta_{2}$ we have $\left|t_{1} T_{\rho}(x)-t_{2} T_{\rho}(y)\right|<\delta_{1}$ for all $\rho>0$.

Let $\delta=\min \left\{\delta_{1}, \delta_{2}\right\}$ and $t \in[0,1]$. For all $\left|t_{1}-t\right|<\delta$ and all $y \in \mathcal{A}$ such that $d(x, y)<\delta$ we have

$$
d\left(h_{\rho}(t, x), h_{\rho}\left(t_{1}, y\right)\right)=d\left(\varphi\left(t T_{\rho}(x), x\right), \varphi\left(t_{1} T_{\rho}(y), y\right)\right)<\epsilon
$$

because $\left|t T_{\rho}(x)-t_{1} T_{\rho}(y)\right|<\delta_{1}$.

By the Ascoli-Arzela theorem given in [11, Theorem XII. 6.4], it follows that every sequence in $\mathcal{F}$ has a subsequence that converges uniformly on compact subsets of $[0,1] \times \mathcal{A}$. In particular, there exists a sequence $\rho_{n} \rightarrow 0$ such that the sequence $\left\{h_{\rho_{n}}\right\}_{n \in \mathbb{N}}$ converges uniformly on compact subsets of $[0,1] \times \mathcal{A}$. Uniform convergence on compact subsets implies that the limit function $h$ : $[0,1] \times \mathcal{A} \rightarrow \mathcal{A}$ is continuous. Moreover, $h$ has the following properties for all $x \in \mathcal{A}, t \in[0,1]$ and $k \in \mathcal{K}$,

$$
\begin{aligned}
& h(0, x)=\lim _{n \rightarrow+\infty} h_{\rho_{n}}(0, x)=\lim _{n \rightarrow+\infty} x=x, \\
& h(1, x)=\lim _{n \rightarrow+\infty} h_{\rho_{n}}(1, x) \in \bigcap_{n=1}^{\infty} \mathcal{U}_{\rho_{n}}=\mathcal{K}, \\
& h(t, k)=\lim _{n \rightarrow+\infty} h_{\rho_{n}}(t, x)=\lim _{n \rightarrow+\infty} x=x .
\end{aligned}
$$

Thus $\mathcal{K}$ is a strong neighborhood deformation retract of $\mathcal{A}$.

Remark 12. Proposition 11 is a theoretical result. It can not be used in practice due to the fact that, in general, we have no information about the semi flow and the functions $T_{\rho}$.

\section{Concluding remarks}

In this paper, we prove some necessary conditions for asymptotic stability of an invariant compact set. A Lyapunov function is used to design a retraction. The main result generalizes a well know theorem of contractibility given by $\mathrm{E}$. Sontag to invariant set by using different topological notions on retractions. 


\section{Acknowledgment}

The first author is extremely grateful to François Laudenbach from the department of Mathematics of the University of Nantes for the many discussions he has had about the notion of asymptotic stability in topology.

\section{References}

[1] E. D. Sontag, Mathematical Control Theory: Deterministic Finite Dimensional Systems, no. 6, Textbooks in Applied Mathematics, Springer-Verlag, New-York, 1998.

[2] S. P. Bhat, D. S. Bernstein, A topological obstruction to continuous global stabilization of rotational motion and the unwinding phenomenon, Systems Control Lett. 39 (1) (2000) 63-70.

[3] N. Bhatia, G. Szegö, Stability Theory of Dynamical Systems, Classics in Mathematics, Springer, 2002.

[4] N. Bhatia, O. Hajek, Local semi-dynamical systems, Lecture Notes in Mathematics, vol. 90, Springer, 1969.

[5] S. T. Hu, Theory of retracts, Wayne State University Press, Detroit, 1965.

[6] E. Bredon, Topology and Geometry, Graduate Texts in Mathematics, vol. 139, Spinger, 1993.

[7] A. Hatcher, Algebraic Topology, Cambridge University Press, 2001.

[8] S. Lang, Fundamentals of Differential Geometry, Springer, 1999.

[9] J. H. Kwak, J. Lee, Retracts in equiconnected spaces, Internat. J. Math. Math. Sci. 16 (1) (1993) 199-200.

[10] B. Cornet, M. Czarneckib, Existence of generalized equilibria, Nonlinear Anal. 44 (5) (2001) 555-574.

[11] J. Dugundji, Topology, Wm. C. Brown, 1989. 